\title{
EFECTOS DE LA SUPRESIÓN DE PENSAMIENTOS NEUTROS Y PENSAMIENTOS INTRUSOS ANÁLOGOS A LAS OBSESIONES EN SUJETOS NORMALES
}

\author{
AMPARO BELLOCH y CARMEN MORILLO \\ Universidad de Valencia
}

(Aceptado en julio de 2002)

\begin{abstract}
Las recientes teorías cognitivo-conductuales sobre el Trastorno Obsesivo-Compulsivo (TOC) postulan que en la génesis de las obsesiones clínicas se producen intentos deliberados de suprimir de la mente pensamientos intrusos indeseados. En este trabajo se presentan los resultados de un estudio experimental sobre la supresión de pensamientos neutros y de pensamientos intrusos de contenido análogo a las obsesiones. Treinta y ocho estudiantes universitarios participaron en un experimento que tenia tres fases: 1) Registro línea-base, 2) Instrucción experimental, y 3) Registro. En cada una se midió la frecuencia de un pensamiento neutro ("oso blanco») o de un pensamiento intruso, personalmente relevante. Todos fueron asignados aleatoriamente a uno de los cuatro grupos siguientes: supresión/neutro, supresión/intruso, control/neutro, y control/intruso. La mitad de los sujetos recibió instrucciones de suprimir el pensamientoobjetivo durante el periodo 2, mientras que la otra mitad recibió instrucciones de simplemente registrar su aparición. Los resultados muestran que en ninguno de los grupos experimentales se produjo un aumento de la frecuencia del pensamiento-objetivo en relación con la línea-base. Sin embargo, las comparaciones entre-grupos revelaron que el grupo de supresión del pensamiento intruso experimentó un mayor número de ocurrencias en el periodo 3, que el grupo que simplemente registraba dicho pensamiento. Los datos sugieren la existencia de un efecto de aumento demorado («efecto rebote») en el caso de los pensamientos intrusos sometidos a esfuerzos de supresión.
\end{abstract}

Palabras clave: Pensamientos intrusos, supresión del pensamiento, trastorno obsesivocompulsivo, obsesiones.

\section{Effects of suppressing neutral and obsession-like thoughts in normal subjects}

Recent cognitive-behavioral theories on obsessive-compulsive disorder (OCD) show that deliberate attempts to suppress intrusive and undesirable thoughts lie at the genesis of clinical obsessions. In this paper the results of an experimental study on the suppression of neutral and obsession-like thoughts in normal subjects are presented. Thirty-eight university students performed in three experimental periods: 1) base-line monitoring, 2) experimental instruction, and 3) monitoring. For each of these periods, the frequency of the occurrence of a "white bear» thought or a personally relevant intrusive thought was registered. Half of the subjects received instructions to suppress the target-thought in period 2, and the other half were instructed to only monitor the target-thought in each of the experimental periods. The results show that none of the experimental groups increased the frequency of their target-thought in relation to the base-line. However, the between-groups comparisons revealed that the suppress/intrusive group registered more intrusions in period 3 than the non-suppress/intrusive group. These data suggest the existence of a rebound effect for intrusive thoughts, whereas this effect was not observed when using neutral thoughts.

Key words: Intrusive thoughts, thought suppression, obsessive-compulsive disorders, obsessions.

Correspondencia: Prof. Amparo Belloch, Departamento de Personalidad Evaluación y Tratamientos Psicológicos, Facultad de Psicología, Avda. Blasco Ibáñez 21, 46010 Valencia. Correo-e: Amparo.Belloch@uv.es

Nota: Este trabajo ha sido parcialmente financiado por el Ministerio de Ciencia y Tecnología (BSO2002-02330).

\section{INTRODUCCIÓN}

Una de las características centrales del trastorno obsesivo-compulsivo (TOC) es la intrusión incontrolable de pensamien- 
tos egodistónicos, recurrentes, e indeseados, es decir obsesiones. La persona afectada intenta controlar estos pensamientos y eliminarlos del flujo de su pensamiento consciente. Las teorías cognitivo-conductuales actuales sobre el TOC hacen una referencia explícita a las estrategias voluntarias que emplean los pacientes para controlar y suprimir sus obsesiones: se considera que estas estrategias voluntarias juegan un papel importante en el mantenimiento y exacerbación de las obsesiones, estando incluidas dentro del concepto más amplio de «neutralización» (p.ej., Salkovskis 1985, 1989; Rachman 1997, 1998; Purdon y Clark, 1999). Según estos planteamientos, los intentos por neutralizar un pensamiento se derivan de interpretaciones o valoraciones disfuncionales de ese pensamiento, pero no están relacionadas con su contenido anormal o extraño, dado que la mayoría de los contenidos de las obsesiones se registran asimismo en la población general sana (Freeston, Ladoucer, Gagnon y Thibodeau, 1991; Freeston y Ladoucer, 1993; Purdon y Clark, 1993, 1994). Por tanto, las valoraciones que realizan los sujetos acerca de sus pensamientos (p.ej. significado personal catastrófico) son las responsables de generar esfuerzos por controlarlos. Sin embargo, estos esfuerzos son habitualmente infructuosos y contribuyen a aumentar la frecuencia y la saliencia del pensamiento. Además, Purdon y Clark (1999) enfatizan el papel que tienen las creencias meta-cognitiEAV sobre la importancia o la necesidad de controlar ciertos pensamientos.

En la última década se han publicado varios estudios que investigan el efecto que producen los esfuerzos por suprimir voluntariamente un pensamiento ("pensamiento-objetivo»), en un contexto de laboratorio. La mayoría de tales estudios se basan en el experimento pionero de Wegner, Schneider, Carter y White (1987), en el que los participantes recibían ins- trucciones bien de suprimir, o bien de expresar, pensamientos sobre un "oso blanco». El experimento típico compara las recurrencias de un pensamiento-objetivo entre dos grupos de sujetos: uno de ellos recibe instrucciones de suprimirlo, mientras que el otro no las recibe. El interés se sitúa en la observación de eventuales incrementos de la frecuencia del pensamiento-objetivo, que muestren un efecto de incremento inmediato, o bien un efecto de incremento demorado. En el primer caso, los esfuerzos de supresión están todavía en marcha, mientras que en el segundo caso estos esfuerzos ya no están presentes, por lo que tal efecto se cataloga como «efecto rebote». A partir de los resultados obtenidos en su experimento original, Wegner (1994) desarrolló un modelo teórico para explicar el fracaso de los intentos voluntarios por controlar ciertos procesos o contenidos mentales, como suprimir un pensamiento o concentrarse activamente en una tarea. Según el modelo, en el intento de suprimir un pensamiento intervienen dos procesos que funcionan simultáneamente: En primer lugar, un "proceso intencional» que realiza una búsqueda consciente y controlada de pensamientos distractores para reemplazar el pensamiento-objetivo; $\mathrm{y}$, en segundo lugar, un "proceso de monitorización paradójica», que es de naturaleza automática y no consciente, y que rastrea los fallos producidos a través del proceso de supresión, buscando continuamente posibles indicios del pensamiento que se pretende suprimir. Además, los pensamientos distractores generados por el primer proceso, se convierten posteriormente en claves que elicitan paradójicamente el pensamientoobjetivo, dado que se asocian con el mismo.

El balance entre ambos procesos (también denominados "búsqueda controlada de distractor» y "búsqueda automática de distractor», respectivamente i.e, 
Purdon 1999) es responsable, según Wegner, de que se produzcan los efectos paradójicos de incremento del pensamiento cuanto se ponen en marcha intentos de supresión intensos. Esto sucede especialmente bajo ciertas condiciones, por ejemplo, cuando existen demandas atencionales que interfieren con el proceso de búsqueda de distractores, o cuando los pensamientos que se pretende suprimir son congruentes con el estado de ánimo.

Revisiones recientes de los estudios experimentales sobre la supresión de pensamientos (p.ej. Purdon, 1999; Purdon y Clark, 2000; Abramowitz, Tolin y Street, 2001) muestran, no obstante, un panorama bastante confuso dadas las considerables inconsistencias de los diferentes resultados obtenidos en diversos estudios. Estas revisiones indican la existencia de importantes diferencias metodológicas, que dificultan comparar adecuadamente los estudios: por ejemplo, diferencias en cuanto al tipo de diseño empleado o a las instrucciones que se proporcionan a los sujetos, o incluso al tipo de pensamiento-objetivo que se utiliza. Además, en el caso de la aplicación de los resultados obtenidos con estos diseños a la comprensión de los mecanismos implicados en el TOC, Purdon y Clark (2000) enfatizan los problemas de validez ecológica que presentan muchos trabajos y la necesidad de utilizar pensamientos intrusos, personalmente relevantes, que motiven de forma natural a los sujetos a poner en marcha intentos de supresión.

Otro punto importante a considerar es que la mayoría de los estudios de supresión se han centrado en la búsqueda de aumentos paradójicos en la frecuencia del pensamiento. Sin embargo, solamente algunos autores han incluido de un modo consistente en sus trabajos otras variables dependientes, tales como el malestar o desagrado asociado a la intrusión, las valoraciones emocionales de los pensamientos o el estado de ánimo. Este es el caso de Trinder y Salkovskis (1994), quienes encontraron evidencias de un incremento del malestar en los sujetos sometidos a instrucciones de supresión durante un periodo de 4 días fuera del laboratorio. Y, más recientemente, Purdon y Clark (1999) han sugerido que, en el caso de los pensamientos intrusos de tipo obsesivo, los efectos más destacables de la supresión quizá no sean los relativos al incremento de frecuencia, sino que tengan que ver más bien con variables como las mencionadas antes. De hecho, ellos encontraron un aumento del malestar y un empeoramiento del estado de ánimo, asociados a la supresión de pensamientos análogos a las obsesiones (Purdon y Clark, 2001).

El presente trabajo se enmarca en esta última línea de investigación. Su propósito es contribuir a comprender mejor los complejos efectos asociados a la supresión de pensamientos intrusos análogos a las obsesiones y personalmente relevantes, en comparación con la supresión de pensamientos neutros e impersonales. En el diseño del protocolo experimental, se han tenido en cuenta las recomendaciones metodológicas de Purdon y Clark (2000). Además, se ha incluido un periodo inicial de línea base, con el objetivo de observar los posibles incrementos intra-grupo de la frecuencia del pensamiento-objetivo asociados a las instrucciones de supresion.

Los principales objetivos de este trabajo han sido averiguar si los intentos deliberados de suprimir dos pensamientoobjetivo diferentes (neutro e intruso personalmente relevante):

a) Producen incrementos paradojicos de la frecuencia en ambos tipos de pensamientos.

b) Producen malestar o desagrado en los sujetos cuando no es exitosa, es decir, cuando a pesar de los esfuer- 
zos de supresión, el pensamientoobjetivo reaparece.

c) Producen cambios significativos en las valoraciones cognitiEAV de los pensamientos intrusos análogos a las obsesiones, cuando tales pensamientos se convierten en objeto de supresión deliberada.

\section{MÉTODO}

\section{Participantes}

Los participantes fueron 38 estudiantes de Psicología (34 mujeres y 4 hombres) con una edad media de 22 años (rango 20-36 años). Todos eran solteros y con un nivel socioeconómico medio. Los sujetos fueron asignados aleatoriamente a uno de los cuatro grupos siguientes: supresión pensamiento neutro $(n=10)$, supresión pensamiento intruso $(n=9)$, control pensamiento neutro $(n=10)$, y control pensamiento intruso $(n=9)$. Los grupos no diferían significativamente en edad ni en nivel socioeconómico. Todos los sujetos dieron su consentimiento explícito para participar en el estudio.

\section{Medidas}

Inventario revisado de Intrusiones ObsesiEAV (Revised Obsessive Intrusions Inventory (ROII; Purdon y Clark 1993,1994): Es un inventario auto-informado que tiene dos partes. La primera contiene 52 items diseñados para evaluar la frecuencia de aparición de pensamientos, imágenes o impulsos intrusos, en una escala de siete puntos, desde 0 («Nunca he tenido este pensamiento») hasta 6 ( Tengo este pensamiento frecuentemente durante todo el día"). En la segunda parte, se pide a los sujetos que examinen sus respuestas a los items, y que teniendo en cuenta el listado ante- rior, seleccionen el pensamiento intruso más desagradable o molesto que han experimentado; posteriormente se les pide que evalúen ese pensamiento a través de 10 dimensiones valoratiEAV (molestia, culpa, preocupación por las consecuencias, dificultad para controlar o eliminar, inaceptabilidad, probabilidad estimada de que pueda ocurrir en la vida real, importancia de controlar, amenaza, responsabilidad y evitación de situaciones desencadenantes de la intrusión), utilizando escalas de 5 puntos desde «En absoluto» hasta "Extremadamente». Después de cumplimentar estas valoraciones, se presenta al sujeto una lista de 10 estrategias de control y se le pide que indique en qué medida utiliza cada una de esas estrategias en respuesta a su pensamiento intruso más desagradable (escalas de 5 puntos desde "Nunca" hasta «Siempre»). Se utilizó la adaptación española del ROII (Morillo, Lucero, Carrió, Cabedo y Belloch, 2001). El pensamiento intruso escogido por cada sujeto fue posteriormente utilizado como pensamiento-objetivo durante el experimento. En el presente estudio, los dos pensamientos intrusos más frecuentemente escogidos como el más desagradable fueron: "...Haberme dejado encendido el fuego de la cocina, la calefacción, la estufa o las luces, y que eso pueda provocar un incendio» (Seleccionado por el $27 \%$ de los sujetos), $y$ "Cuando estoy en un lugar alto.... saltar al vacío» (Seleccionado por $12 \%$ de los sujetos)

\section{Maudsley Obsessive Compulsive} Inventory (MOCI; Hodgson y Rachman, 1977). Cuestionario auto-informado de 30 items, con respuestas verdadero/falso, muy frecuentemente utilizado en estudios con sujetos normales y clínicos para evaluar la presencia de síntomas obsesivo-compulsivos. Las puntuaciones totales del MOCI se sitúan en un rango entre 0 y 30. 
Inventario de Depresión de Beck (BDI; Beck, Rush, Shaw y Emery, 1979): Autoinforme de 21 items, ampliamente utilizado y validado, que evalúa la presencia de sintomatología depresiva. Los sujetos evalúan la gravedad de 21 síntomas depresivos utilizando escalas de cuatro puntos desde 0 (Síntoma no presente) hasta 3: (Síntoma muy intenso). Las puntuaciones totales en el BDI se sitúan en un rango entre 0 y 63.

Cuestionario de Ansiedad Estado-Rasgo (STAI E-R; Spielberger, Gorsuch, Lushene, Vagg y Jacobs, 1983): Medida autoinformada de ansiedad general consistente en 40 items. Los primeros 20 (STAI-E) evalúan ansiedad-estado, referidos a cómo el sujeto se siente en ese momento. Los siguientes 20 (STAI-R) evalúan ansiedadrasgo, referidos a cómo se siente el sujeto generalmente. En el presente trabajo se utilizó sólo la escala de ansiedad-estado.

Registro de frecuencia del pensamiento objetivo: Con el objetivo de registrar la frecuencia de ocurrencia (número de intrusiones) del pensamiento objetivo en cada uno de los periodos experimentales, los sujetos tenían que realizar una marca en un espacio delimitado de una hoja de papel cada vez que ocurría (o les venía a la mente) el pensamiento-objetivo.

Escalas Análogo Visuales (EAV): A lo largo del experimento se tomaron cuatro medidas, diseñadas expresamente para este estudio. Todas estas variables fueron evaluadas con escalas análogo-visuales (EAV) de $10 \mathrm{~cm}$. graduadas de 0 a 10.

a) Estimación del tiempo pensando en el pensamiento-objetivo (EAV de Tiempo desde $0=$ «En ningún momento» hasta $10=$ «Durante todo el tiempon). Todos los sujetos cumplimentaron esta escala después de los tres periodos experimentales. b) Esfuerzo de supresión: Después de los periodos 2 y 3 se les preguntó a los sujetos: en qué medida se habían esforzado en quitarse de la cabeza el pensamiento durante el intervalo correspondiente (EAV de Esfuerzo desde 0: «Nada en absoluto" hasta 10: "Muchísimo". Esta medida tenía un doble propósito: en primer lugar, comprobar que el sujeto segura las instrucciones de supresión y, en segundo lugar, evaluar la tendencia a suprimir espontáneamente en ausencia de instrucciones explícitas para hacerlo.

c) Exito de la supresión: «¿En qué medida has conseguido NO pensar en pensamiento objetivo- durante este último periodo?». (EAV de Exito desde 0: «No lo he conseguido en absoluto» hasta 10: "Lo he conseguido del todo"). Con esta pregunta obtuvimos una estimación subjetiva del éxito de la supresión, que podría no estar relacionada con la tasa real de intrusiones durante la fase de supresión. Esta medida la cumplimentaron solamente los sujetos incluidos en los grupos de supresión, después del periodo 2 del experimento.

d) Molestia causada por el pensamiento-objetivo: Después de los periodos 2 y 3 del experimento, se les preguntó a los sujetos: «¿En qué medida te ha resultado molesto no poder evitar que te viniera a la mente el pensamiento durante estos cinco minutos?». (EAV de Molestia desde 0: «Nada molesto» hasta 10: «Extremadamente molesto"). Esta medida resultaba interesante para investigar un aspecto importante de la reacción emocional a la experiencia de supresión y la dificultad para eliminar un pensamiento-objetivo.

Valoraciones de los pensamientosobjetivo intrusos: Además, los sujetos que 
tenían el pensamiento intruso como pensamiento-objetivo en el experimento respondieron a tres preguntas adicionales después de haber finalizado el experimento: malestar, culpa y preocupación por las consecuencias de tener el pensamiento (Desde 0: "Nada en absoluto" hasta 4: «Extremadamente». Estas dimensiones valorativas son idénticas a algunas de las preguntas de la segunda parte del ROII, completado por los sujetos una semana antes. Por ello, dispusimos de valoraciones pre y post-experimentales de los pensamientos intrusos.

\section{Procedimiento}

En una sesión de evaluación inicial, 53 estudiantes de Psicología cumplimentaron los cuestionarios antes mencionados, precedidos de una hoja en la que se recogían los datos demográficos así como la posible existencia de trastornos psicológicos, enfermedades relevantes, y tratamientos recientes. Se excluyeron aquellos sujetos que referían problemas psicológicos, y también aquellos que presentaban puntuaciones altas en los cuestionarios siguientes: $\mathrm{BDI}>10, \mathrm{STAI}-\mathrm{E}>30$, y/o $\mathrm{MOCI}>8$. Asimismo, se controló que la frecuencia de aparición del pensamiento intruso escogido del ROII no fuera superior a 4 ( $\mathrm{A}$ menudo, tengo este pensamiento: una o dos veces por semana») y que las puntuaciones en valoraciones no fueran de 4 («Extremadamente») en dos o más dimensiones valorativas. Una vez aplicados estos criterios de exclusión, solamente incluimos en el experimento aquellos sujetos cuyo pensamiento escogido del ROII tuviera al menos una frecuencia de 2 ( Ocasionalmente, tengo este pensamiento unas pocas de veces al año»), y que refirieran al menos una puntuación de 2 («Algo molesto») en la valoración de molestia asociada a ese pensamiento. Los 38 suje- tos finalmente seleccionados fueron citados para realizar el experimento una semana después.

El experimento tuvo lugar en grupos de cinco personas en una habitación silenciosa, bien iluminada, y sin ventanas. Los participantes en cada sesión se sentaron separados unos de otros, y se les entregó a cada uno de los participantes el protocolo experimental en forma de bloque de cuartillas grapadas, un magnetófono con auriculares que contenía las instrucciones grabadas y un rotulador rojo. Como introducción al experimento únicamente se les decía que el propósito del experimento era investigar algunos procesos de pensamiento.

Se asignó a los sujetos sus pensamientos-objetivo: En el caso de los grupos de pensamiento neutro, se pedía a los participantes que imaginaran un oso blanco durante un periodo de un minuto. En el caso de los grupos de pensamiento intruso (obsesivo), se mostró a los participantes su ROII cumplimentado la semana previa y se les pedía que identificaran su pensamiento intruso más problemático, seleccionado a través de este instrumento. Entonces tenían que resumirlo en una frase breve, personalmente significativa, y tenían que indicar si tenía forma de imagen, impulso o pensamiento; después se les pedía que pensaran (o imaginaran) durante un minuto su pensamiento personalmente relevante.

Posteriormente a esta fase de "priming", se informó a los sujetos de lo siguiente: «Durante este experimento, se te pedirá que registres las ocurrencias del pensamiento-objetivo realizando marcas rojas sobre el papel cada vez que el pensamiento te venga a la mente». Los sujetos practicaban el registrar las ocurrencias del pensamiento de este modo con los ojos cerrados.

A continuación se colocaban los auriculares, escuchaban las instrucciones grabadas, y se sometían a tres periodos 
experimentales de cinco minutos cada uno. Durante cada uno de estos periodos, los sujetos permanecían registrando las ocurrencias del pensamiento-objetivo con los ojos cerrados y en absoluto silencio, con los auriculares colocados. Una señal acústica marcaba el principio y el final de cada periodo. Las instrucciones específicas para cada grupo y periodo fueron similares a las señaladas por Salkovskis y Campbell (1994), teniendo asimismo en cuenta las recomendaciones de Purdon y Clark (2000):

Periodo 1: Se trata de un periodo de línea base, utilizado para evaluar la frecuencia inicial del pensamiento-objetivo. Todos los sujetos recibieron instrucciones de a...Puedes pensar en cualquier cosa incluido el pensamiento-obje- tivo, pero no trates de suprimir ningún pensamiento...».

Periodo 2. La mitad de los sujetos (grupos 1 y 2) recibio instrucciones de suprimir el pensamiento (... es muy importante que trates 10 más fuertemente que puedas de NO pensar en (oso blanco / pensamiento intruso), que trates de suprimir ese pensamiento de tu mente. Puedes pensar en cualquier cosa excepto en (oso blanco/ pensamiento intruso)....) mientras continuaban registrando el pensamiento. El resto de sujetos (grupos 3 y 4) recibió instrucciones similares a las de línea base.

Periodo 3. Todos los grupos recibieron instrucciones similares a las utilizadas en la línea base.

La Figura 1 representa el diseño general del experimento, que en líneas generales sigue el esquema propuesto por Abramowitz y cols. (2001).

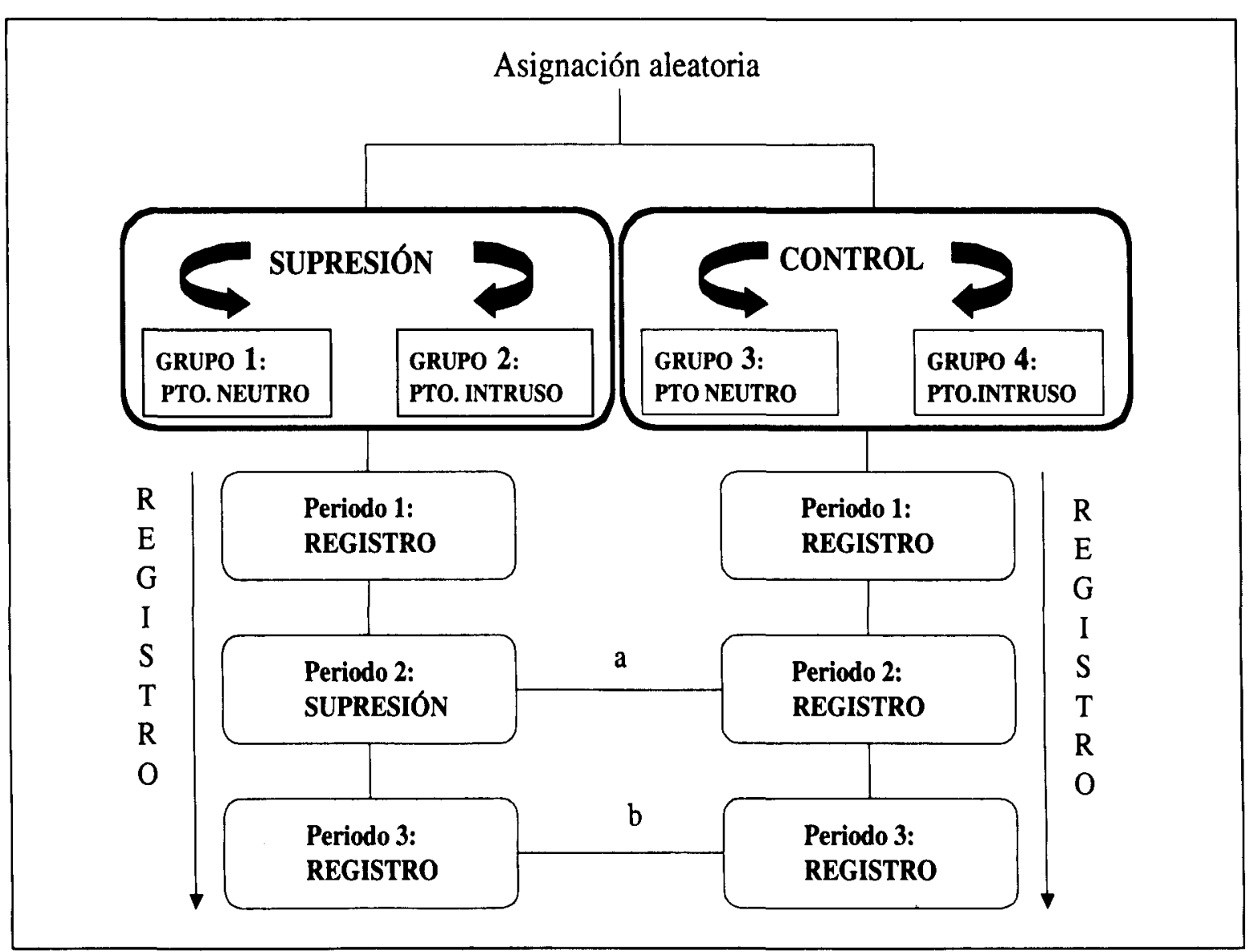

Figura 1. Diseño del experimento de supresión con los dos tipos de pensamiento-objetivo (figura basada en Abramowitz y cols, 2001). El periodo 1 constituye la línea base. Las letras (a) y (b) representan las comparaciones apropiadas para medir cada efecto en relación a los grupos control (efecto de aumento inmediato y efecto rebote, respectivamente). 


\section{RESULTADOS}

\section{Análisis preliminares}

No se detectaron diferencias significativas pre-experimentales entre los grupos en las puntuaciones obtenidas en los cuestionarios (ROII, BDI, MOCI y STAI-E) ni en las valoraciones del pensamiento intruso detectado mediante el ROII, lo que indica una correcta asignación aleatoria a los grupos. En la Tabla 1 se muestran las medias y desviaciones típicas pertenecientes a cada grupo en estas medidas, así como los valores de $F$ para cada comparación.

A continuación se realizó un análisis de varianza para determinar si los sujetos siguieron las instrucciones de suprimir o no suprimir. En la Tabla 2 se presentan las puntuaciones medias y desviaciones típicas de los grupos experimentales en el esfuerzo de supresión del pensamientoobjetivo durante los periodos 2 y 3 . Se llevó a cabo un ANOVA factorial mixto 2 (Pensamiento objetivo: neutro vs. intruso) $\times 2$ (Tipo de instrucción: supresión vs. control) $\times 2$ (Periodos 1 y 2 , variable intrasujetos), que mostró efectos principales significativos para Pensamiento objetivo $\left(F_{1,34}=11,25, p<0,01\right)$, Tipo de instrucción $\left(F_{1,34}=11,25, p<0,01\right)$ y para Periodo $\left(F_{1,34}=\right.$ $60,71, p<0,001)$. Además, resultaron significativas las interacciones Pensamientoobjetivo $\times$ Periodo $\left(F_{1,34}=8,24, p<0,01\right)$, y Tipo de instrucción $\times$ Periodo $\left(F_{1,34}=13,22\right.$, $p<0,01)$. De acuerdo con estos análisis, los grupos de supresión/neutro y supresión/intruso realizaron mayores esfuerzos de supresión durante el periodo 2 que los dos grupos control.

Finalmente, con el fin de averiguar si las dos variables dependientes utilizadas para cuantificar el pensamiento objetivo (número de marcas y tiempo estimado), eran equiparables, se realizaron correlaciones Pearson entre ambas, para cada uno de los tres periodos experimentales. Los coeficientes obtenidos fueron 0,57 , 0,75 , y 0,74 , para los períodos 1,2 y 3 respectivamente $(p<0,0001)$. Ambas medidas

Tabla 1. Puntuaciones en los cuestionarios y en las valoraciones del pensamiento intruso más molesto

\begin{tabular}{lrrrrrr}
\hline \multicolumn{1}{c}{ Medidas } & $\begin{array}{c}\text { Grupo 1: } \\
\text { Supresión } \\
\text { Neutro } \\
(n=10)\end{array}$ & $\begin{array}{c}\text { Grupo 2: } \\
\text { Supresión } \\
\text { Intruso } \\
(n=10)\end{array}$ & $\begin{array}{c}\text { Grupo 3: } \\
\text { Control } \\
\text { Neutro } \\
(n=10)\end{array}$ & $\begin{array}{c}\text { Grupo 4: } \\
\text { Control } \\
\text { Intruso } \\
(n=10)\end{array}$ & $F_{3,36}$ & $P_{<}$ \\
\hline ROII & $14,70(6,63)$ & $29,20(22,26)$ & $20,30(10,23)$ & $21,70(21,48)$ & 1,27 & 0,30 \\
BDI & $2,80(2,86)$ & $2,90(2,28)$ & $3,60(3,13)$ & $2,60(2,67)$ & 0,25 & 0,86 \\
MOCI & $2,10(1,37)$ & $\mathbf{3 , 5 0}(2,51)$ & $3,30(2,54)$ & $3,30(2,75)$ & 0,74 & 0,54 \\
STAI-E & $12,70(6,70)$ & $13,30(8,58)$ & $20,30(10,37)$ & $11,70(8,98)$ & 2,01 & 0,13 \\
Molestia & $1,90(0,99)$ & $1,50(0,85)$ & $1,50(0,97)$ & $1,40(0,70)$ & 0,63 & 0,60 \\
Culpa & $1,10(1,37)$ & $0,40(0,52)$ & $0,70(0,48)$ & $0,60(0,70)$ & 1,21 & 0,32 \\
Preocupación por & $1,30(1,34)$ & $1,00(0,82)$ & $1,00(0,67)$ & $1,70(1,42)$ & 0,90 & 0,45 \\
por consecuencias & & & &
\end{tabular}

Nota: Los datos están expresados como: Media (Desviación típica)

Tabla 2. Valoraciones del esfuerzo de supresión

\begin{tabular}{lrcc}
\hline Grupos & $n$ & Periodo 2 & Periodo 3 \\
\hline Supresión & & & \\
$\quad$ Neutro & 10 & $6,20(2,25)$ & $1,90(2,13)$ \\
Intruso & 9 & $4,33(2,18)$ & $1,67(1,32)$ \\
Control & & & \\
$\quad$ Neutro & 10 & $4,10(2,73)$ & $1,90(1,52)$ \\
Intruso & 9 & $0,67(1,12)$ & $0,33(0,71)$ \\
\hline
\end{tabular}

Nota: Los datos están expresados como: Media (Desviación típica) 
Tabla 3. Frecuencia del pensamiento-objetivo

\begin{tabular}{llrll}
\hline Grupos & $n$ & Periodo 1 & Periodo 2 & Periodo 3 \\
\hline Supresión & & & & \\
Neutro & 8 & $11,25(5,50)$ & $8,00(6,00)$ & $6,75(3,65)$ \\
Intruso & 9 & $6,33(2,18)$ & $4,22(2,77)$ & $5,44(2,07)$ \\
Control & & & & \\
$\quad$ Neutro & 9 & $11,56(5,73)$ & $9,22(4,87)$ & $7,11(4,04)$ \\
Intruso & 9 & $4,78(1,86)$ & $1,56(0,88)$ & $0,78(0.97)$ \\
\hline
\end{tabular}

Nota: Los datos están expresados como: Media (Desviación típica).

de las ocurrencias del pensamiento-objetivo resultaron, por tanto, equiparables, lo cual nos permitió utilizar una sola de ellas para el posterior análisis de frecuencia.

Efectos de la supresión sobre la tasa de pensamientos

Se eliminaron inicialmente tres sujetos por presentar tasas extremas en la variable frecuencia (el número de marcas era superior o igual a tres desviaciones típicas por encima de la media grupal durante uno o más periodos), y se transformaron los datos (mediante raíz cuadrada) para ajustarlos a la distribución normal. En la Tabla 3 se muestran las puntuaciones medias y desviaciones típicas de los cuatro grupos experimentales a través de los tres periodos.

Se realizó un ANOVA factorial mixto 2 (Pensamiento-objetivo) $\times 2$ (Tipo de instrucción) $\times 3$ (Periodo variable intra-sujetos), que reveló efectos principales significativos para Pensamiento-objetivo $\left(F_{1,31}=\right.$ 40,02; $p<0,001)$, Tipo de instrucción $\left(F_{1,31}=\right.$ $5,30 ; p<0,05)$, y Periodo $\left(F_{2,30}=15,96\right.$; $p<0,001)$. Asimismo, se obtuvieron efectos significativos en las interacciones de Pensamiento-objetivo $\times$ Tipo de instrucción $\left(F_{1,31}=8,42 ; p<0,01\right)$, y para Tipo de instrucción $\times$ Periodo $\left(F_{2,30}=4,38 ; p<0,05\right)$. La Figura 2 representa los resultados obtenidos en la frecuencia del pensamiento-objetivo.

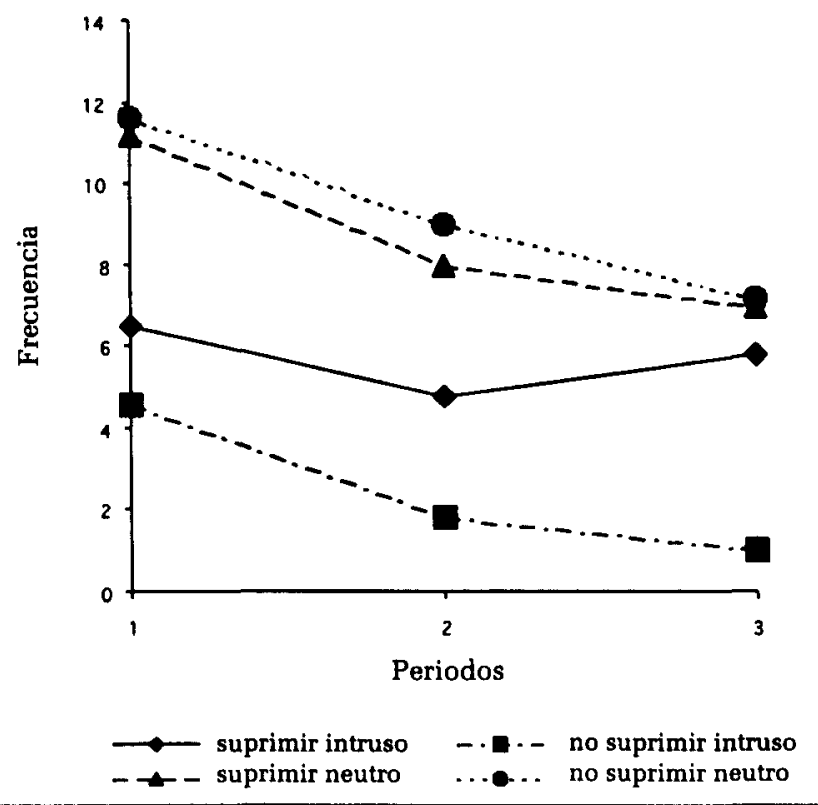

Figura 2. Frecuencia de intrusiones a lo largo de los tres periodos experimentales en los cuatro grupos de sujetos. 
Como muestra la Figura 2, se produjo una disminución en la frecuencia del pensamiento neutro ("oso blanco») a lo largo de los periodos experimentales en los dos grupos de sujetos (registro y supresión). Sin embargo, en el caso de los grupos de pensamiento intruso, la instrucción de supresión produjo un aumento en la frecuencia del pensamiento, en comparación con la instrucción de registro simple (no suprimir).

Dado que los grupos de "oso blanco» registraron mayores frecuencias del pensamiento a lo largo de todo el proceso experimental que los grupos de pensamiento intruso, se realizaron dos ANOVAS mixtos bifactoriales (2: Tipo de instrucción $\times$ 3: Periodo), uno para cada tipo de pensamiento-objetivo, con el objetivo de clarificar más los resultados. En el caso de los grupos de "oso blanco», se obtuvo un efecto principal de Periodo $\left(F_{2,14}=4,51 ; p<0,05\right)$ que revela una disminución de las frecuencias del «oso blanco" del periodo 1 al periodo 3 , sin otros efectos significativos. Estos resultados indican que no existen aumentos de frecuencia debidos a la supresión en los grupos de pensamiento neutro: ni efecto inmediato ni efecto demorado (rebote).

En el caso de los grupos de pensamiento intruso, obtuvimos dos tipos de efectos principales significativos: para Tipo de instrucción $\left(F_{1,16}=22,60 ; p<\right.$ $0,0001)$ y para Periodo $\left(F_{2,16}=14,85\right.$; $p<0,0001)$, además de una interacción significativa: Tipo de instrucción $\times$ Periodo $\left(F_{2,16}=12,34 ; p<0,01\right)$. El análisis de efectos simples reveló que las diferencias inter-grupales eran solamente significativas para el periodo 3 , en el que el grupo de supresión/intruso registró más frecuencias de pensamientoobjetivo que el grupo control/intruso $\left(F_{1.16}=39,14 ; p<0,0001\right)$, pero no se constataron diferencias significativas para los periodos $1\left(F_{1,16}=2,53 ; p<0,132\right)$ y 2
$\left(F_{1,16}=4,39 ; p<0,052\right)$. En consecuencia, estos resultados apoyan un efecto paradójico en la supresión de los pensamientos intrusos, específicamente un efecto de aumento demorado (o «efecto rebote") en el periodo 3 , sin que se evidencie un efecto de aumento inmediato (en el periodo 2). Los efectos diferenciales obtenidos están representados en la Figura 2.

El siguiente análisis se realizó para examinar si los grupos de supresión experimentaban un incremento de la frecuencia del pensamiento-objetivo con respecto a su línea base de frecuencia (número de marcas en el periodo 1). Se calculó para cada grupo de supresión (supresión/neutro y supresión/intruso) el "Índice de rebote» medio para los periodos 2 y 3 (Rutledge, Hancock, y Rutledge, 1996), según la siguiente fórmula: Número de intrusiones durante el periodo experimental / (Número de intrusiones durante el periodo experimental + Número de intrusiones en la línea base). Según los autores mencionados, 0,60 es un punto de corte apropiado para considerar significativo un índice de rebote. Los valores medios de los índices de rebote que obtuvimos fueron los siguientes ( samiento intruso, respectivamente): 0,42 y 0,40 para el periodo $2 ; 0,38$ y 0,46 para el periodo 3. En consecuencia, estos valores indican que no existen incrementos de frecuencia intra-grupo en ningún tipo de pensamiento-objetivo, con respecto a la frecuencia de intrusiones de la línea base.

\section{Molestia causada por la intrusión}

El objetivo del siguiente análisis fue investigar la reacción emocional de los sujetos a la experiencia de supresión, y las consecuentes dificultades para eliminar un pensamiento de la mente. El 
Tabla 4. Molestia causada por la intrusión

\begin{tabular}{lrcc}
\hline Grupos & $n$ & Periodo 2 & Periodo 3 \\
\hline Supresión & & & \\
$\quad$ Neutro & 10 & $3,80(2,78)$ & $1,50(1,84)$ \\
$\quad$ Intruso & 9 & $2,78(2,17)$ & $1,89(1,27)$ \\
Control & & & \\
$\quad$ Neutro & 10 & $3,40(2,22)$ & $2,40(2,17)$ \\
Intruso & 9 & $0,67(0,71)$ & $0,00(0,00)$ \\
\hline
\end{tabular}

Nota: Los datos están expresados como: Media (Desviación típica).

primer análisis se realizó para examinar el éxito subjetivo de los esfuerzos de supresión, tal y como éste fue valorado por los sujetos. Recordemos que esta variable únicamente se midió en los dos grupos de supresión después del segundo periodo experimental, es decir, después de darles explícitamente las instrucción de supresión. No se encontraron diferencias en el éxito subjetivo de supresión estimado entre los dos grupos de pensamiento-objetivo $\left(t_{1,17}=\right.$ $-0,88 ; p=0,39$ ). Por tanto, el grupo de "oso blanco» y el grupo de pensamiento intruso mostraron un éxito percibido similar, y este resultado indica que el éxito subjetivo parece ser independiente del contenido neutro o intruso del pensamiento objetivo.

A continuación analizamos la molestia producida por las ocurrencias del pensamiento objetivo (molestia causada por la intrusión), a pesar de los intentos por suprimirlas. Es importante tener en cuenta que en este caso evaluamos el malestar o molestia asociada a la dificultad para suprimir completamente el pensamientoobjetivo, y que en ningún caso nos referimos al malestar provocado por la intrusión en sí misma. En la Tabla 4 se muestran las puntuaciones medias y desviaciones típicas de los grupos en esta variable, que se evaluó tras los periodos 2 y 3.

Se llevó a cabo un ANOVA factorial mixto 2 (Pensamiento-objetivo) $\times 2$ (Tipo de instrucción) $\times 2$ (Periodos 2 y 3 , factor intra-sujetos), para la molestia causada por la intrusión. Se encontraron efectos principales significativos para Pensamiento-objetivo $\left(F_{1,34}=6,80, p<0,05\right)$ y para Periodo $\left(F_{1,34}=21,33 ; p<0,0001\right)$, así como una interacción significativa de Pensamiento-objetivo $\times$ Tipo de instrucción $\left(F_{1,34}=4,14 ; p<0,05\right)$. Estos resultados se representan en la Figura 3 . El análisis de efectos simples mostró que, en el caso de los pensamientos intrusos, el grupo de supresión valoraba como más molestas las recurrencias del pensamiento-objetivo que el grupo control, y esto ocurría en ambos periodos ( 2 y 3 ). Es decir, cuando se dieron instrucciones de suprimir el pensamiento intruso, las recurrencias de este pensamiento-objetivo se consideraban más molestas, aunque ya no estuvieran presentes las instrucciones explícitas de supresión (que es lo que ocurría en el periodo 3).

Para examinar de otro modo la asociación entre el esfuerzo de supresión y la molestia causada por la intrusión, se calcularon coeficientes de correlación bivariada (Pearson) entre el esfuerzo de supresión (en el periodo 2) y la molestia (en los periodos 2 y 3 ), para los dos tipos de pensamiento-objetivo. Obtuvimos coeficientes de correlación significativos entre ambas variables en los periodos 2 y $3(r=0,60$ y $r=0,56$, respectivamente; $p<0,05$ ), únicamente en los grupos de pensamiento intruso. Estos resultados constituyen una evidencia adicional de la reacción emocional asociada a los intensos esfuerzos por suprimir un pensamiento intruso indeseado. 


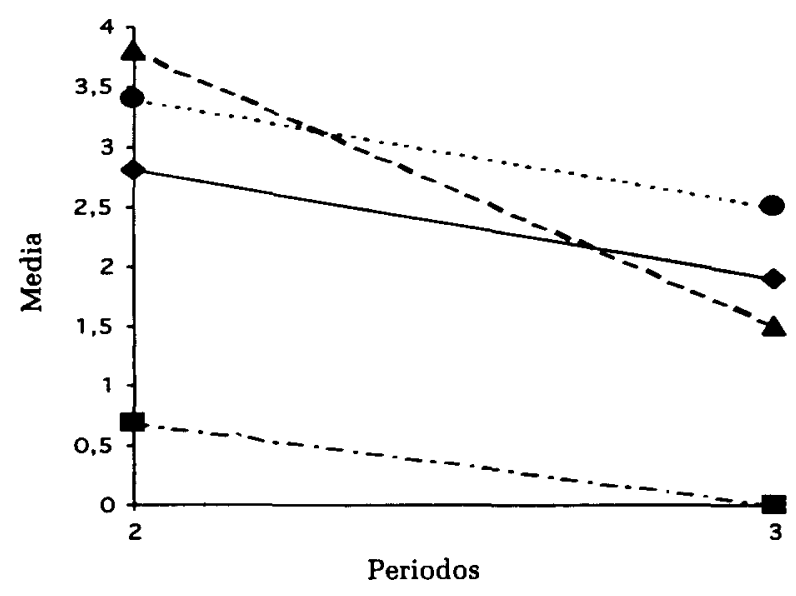

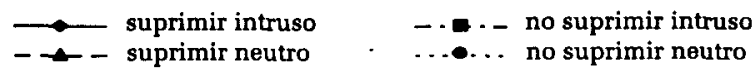

Figura 3. Molestia causada por las intrusiones del pensamiento-objetivo en los cuatro grupos de sujetos (periodos 2 y 3 )

Valoraciones post-experimentales de los pensamientos intrusos

Se realizaron pruebas t para muestras independientes para comparar las valoraciones post-experimentales que realizaban los sujetos de los grupos 2 (supresión/intruso) y 4 (control-intruso) respecto a sus respectivos pensamientosobjetivo intrusos, tras el experimento. Los resultados mostraron que una vez finalizado el experimento, el grupo de supresión/intruso, valoró su pensamiento-objetivo como más molesto o desagradable que el grupo control/intruso $\left(t_{1,16}=3,20 ; p<0,01\right)$. Sin embargo, no hubo diferencias entre ambos grupos en el resto de valoraciones de los pensamientos intrusos medidas. (Culpa: $t_{1,16}=0,72, p=$ 0,48; Preocupación: $t_{1,16}=0,95, p=0,36$ ).

$\mathrm{El}$ análisis intra-grupo (pruebas t para muestras relacionadas) del grupo de supresión/intruso, mostró que valoraban su pensamiento intruso como más desagradable una semana antes del experimento $(1,5 \pm 0,85)$ que inmediatamente después $(1 \pm 0,7)(t=2,37 ; p<0,04)$, pero no se detectaron diferencias pre- vs. postexperimentales en el resto de dimensiones valorativas del pensamiento intruso. Este fue también el caso del grupo de control/intruso, ya que se observaron diferencias pre- vs. post-experimentales en las dimensiones de molestia (preexperimento: $1,3 \pm 0,7$; post: $0,11 \pm 0,33$; $t=5,5 ; p<0,001$ ) y culpa (pre: $0,60 \pm 0,70$; post: $0,11 \pm 0,4 ; t=3,16 ; p<0.01$ ).

\section{DISCUSIÓN}

El objetivo principal de este estudio ha sido contribuir a entender mejor los controvertidos efectos que tienen los esfuerzos voluntarios por suprimir pensamientos sobre el flujo de conciencia posterior. La comprensión de estos efectos puede aplicarse especialmente al TOC, un trastorno mental caracterizado por los esfuerzos deliberados que realizan los pacientes por suprimir sus pensamientos intrusos, es decir, sus obsesiones. 
Los análisis preliminares realizados sobre los datos demográficos, y sobre los cuestionarios de ansiedad, depresión, sintomatología obsesiva, presencia de pensamientos intrusos y valoraciones de los mismos, mostraron que los cuatro grupos de sujetos no diferían en ninguna de estas variables. También verificamos que los sujetos incluidos en los grupos de supresión siguieron las instrucciones adecuadamente. Por otra parte, al observar que las dos variables dependientes (frecuencia y tiempo estimado), diseñadas para cuantificar la ocurrencia de intrusiones a través de los periodos experimentales, estaban significativamente correlacionadas y arrojaban los mismos resultados, decidimos presentar únicamente los resultados obtenidos con la primera de estas dos variables dependientes (que es, por otro lado, la que se suele utilizar en los estudios publicados al respecto).

Nuestro primer objetivo era constatar la presencia de un incremento de la frecuencia del pensamiento-objetivo como consecuencia de los esfuerzos de supresión deliberada. En primer lugar, nuestros resultados sugirieron que este efecto de incremento no se evidenciaba, ya que ninguno de los grupos de supresión mostraba un aumento de intrusiones durante y después del periodo de supresión con respecto a su tasa inicial de intrusiones. Por consiguiente, los esfuerzos intencionales de supresión no conllevan un incremento significativo del número de pensamientos neutros ni de pensamientos intrusos, cuando comparamos este número con el observado en la líneabase. Que nosotras sepamos, existen solamente dos estudios que hayan utilizado un periodo de línea base de registro con el propósito de realizar comparaciones intra-grupos (Rutledge, 1997; Janeck y Calamari, 1999). Nuestros hallazgos coinciden con los obtenidos por los mencionados autores, ya que tampoco encontra- ron efectos de incremento paradójico de la frecuencia de intrusiones al compararla con la línea base de frecuencia.

De todos modos, como se puede observar en la Figura 2, mientras que en los grupos del «oso blanco» y en el grupo control del pensamiento intruso la frecuencia de intrusiones desciende claramente a lo largo de los tres periodos del experimento, el grupo de supresión/intruso, no mostró un descenso tan acusado en la fase 2 (instrucción de supresión) como los otros grupos. Además de ello, en este grupo las diferencias entre la línea base y fase de supresión estuvieron cercanas a la significación estadística $(p<0.052)$. Sin perder de vista esta limitación estadística, este resultado ofrece un apoyo parcial a la sugerencia de Purdon y Clark (2001) de que la instrucción de suprimir intencionadamente un pensamiento interrumpe el proceso natural de habituación a ese pensamiento. Estos autores no detectaron efectos paradójicos en la frecuencia tras la supresión, para ningún tipo de pensamiento objetivo (neutro, positivo, o intruso), pero sí que pudieron constatar que en los grupos de supresión no se producía un descenso tan marcado en la frecuencia de pensamientos como en los de no-supresión. Esta menor disminución en la frecuencia de intrusiones como consecuencia de la instrucción de supresión, es similar a la que nosotras hemos constatado pero, en nuestro caso, sólo con los pensamientos intrusos. En nuestra opinión este resultado resulta congruente con los obtenidos en las comparaciones inter-grupales (i.e., entre los grupos de supresión y los grupos control). Ambos conjuntos de grupos evidenciaron un patrón de resultados muy distinto, atribuible fundamentalmente a las diferencias en el tipo de pensamiento-objetivo (intrusos vs. neutros), ya que únicamente hemos encontrado efectos paradójicos de aumento de la frecuencia (efecto rebote) en sujetos sometidos a la instrucción de suprimir pensamientos intrusos. 
Otros estudios que han utilizado también pensamientos intrusos personalmente relevantes no han encontrado efecto rebote (Roemer y Borkovec, 1994; Mathews y Milroy, 1994; Muris, Merckelbach, Horselenberg, Sijsenaar y Lecuw, 1997; Wang, 1998; McNally y Ricciardi, 1996; Janeck y Calamari, 1999; Purdon y Clark, 2001). Sin embargo, un meta-análisis recientemente publicado (Abramowitz, Tolin y Street (2001), revela que mientras que el tamaño del efecto de aumento inicial es de pequeño a moderado en magnitud, el tamaño del efecto demorado o rebote es positivo, pero de pequeño a moderado en magnitud. Además de esto, cuando ambos efectos eran significativos, los efectos rebote eran mayores que los efectos de aumento inicial. Nuestros resultados coinciden con las conclusiones de este meta-análisis, al menos por lo que se refiere al efecto rebote.

Es el presente estudio no se obtuvo efecto paradójico de aumento de la frecuencia para los pensamientos neutros («oso blanco»). Este resultado es contrario a los procedentes de otros trabajos (p.ej. Wegner, Schneider, Carter y White, 1987; Lavy y Van den Hout, 1990; Clark, Ball y Pape, 1991; Clark, Winton y Thynn, 1993; Bowers y Woody, 1996). Esta discrepancia puede deberse a las diferencias en las instrucciones experimentales dadas a los sujetos incluidos en los grupos control: mientras que los mencionados estudios encontraron un efecto paradójico utilizando un grupo control de expresión del pensamiento-objetivo («intenta pensar en un oso blanco»), nosotras utilizamos instrucciones control que simplemente mencionan el pensamiento-objetivo ("piensa en lo que quieras, puedes pensar en un oso blanco pero no tienes porqué hacerlo"). Algunos autores han puntualizado que estas últimas instrucciones tienen una mayor validez ecológica, y que además no tienen asociados efectos de «ceiling» (p.ej. Abramowitz y cols., 2001). De hecho, cuando se utilizan estas instrucciones-control de mención, los investigadores no encuentran efectos paradojicos cuando utilizan pensamientos-objetivo neutros (Merckelbach, Muris, van der Hout y de Jong, 1991; Muris, Merckelbach y de Jong, 1993; Clark y Purdon, 2001).

El segundo objetivo era investigar la presencia de consecuencias emocionales negativas derivadas de los fracasos en los esfuerzos de supresión. No hubo diferencias entre los grupos de supresión/ neutro y supresión/intruso en cuanto al éxito percibido de la supresión, y ambos grupos mostraron un descenso en la molestia causada por las recurrencias del pensamiento objetivo a lo largo del experimento. Sin embargo, las recurrencias del pensamiento neutro fueron consideradas como más molestas que las del pensamiento intruso y personalmente relevante. Es posible que este resultado refleje simplemente el hecho de que los sujetos están acostumbrados a su pensamiento intruso, así como a sus recurrencias inesperadas, a pesar de los esfuerzos para suprimirlos. Pero en el caso de los pensamientos neutros puede que ocurra todo lo contrario: dada su inocuidad o irrelevancia, el sujeto tiene la expectativa de que puede y debe controlar de un modo eficaz su recurrencia, y cuando lo que sucede es que no tiene éxito, entonces considera la mera reaparición del pensamiento como algo molesto. Esto podría ser una razón del por qué este grupo valora más molestas las recurrencias. Por otro lado, con respecto a los grupos de pensamiento intruso, el grupo de supresión /intruso experimenta las recurrencias del pensamiento-objetivo como más molestas que el grupo de control/intruso. Este hallazgo es consistente con los efectos egodistónicos que tienen los fracasos de pacientes obsesivos en el control de las recurrencias de sus 
obsesiones. Además, algunos estudios han mostrado también que los pensamientos intrusos son más difíciles de suprimir que los pensamientos neutros (p.ej. Mc Nally y Ricciardi, 1996; Purdon y Clark, 2001).

Por último, y en relación con nuestro tercer objetivo, los esfuerzos de supresion produjeron un incremento del malestar asociado al pensamiento intruso, como puede observarse al inspeccionar las diferencias en esta dimensión valorativa entre los dos grupos de pensamiento intruso (supresión/intruso y control/intruso), después de la sesión experimental. Sin embargo, los resultados deben ser considerados con cautela dado que las comparaciones intra-grupo mostraron un descenso de la molestia causada por el pensamiento intruso después del experimento, en ambos grupos de pensamiento intruso. A pesar de ello, es particularmente interesante que el decremento preversus post- experimento fuera claramente acusado en el grupo control, mientras que el grupo de supresión mostrara solamente una disminución pequeña. De nuevo, al igual que en el caso de la disminución de la frecuencia, parece que los esfuerzos deliberados de supresión interfieren con la habituación a las intrusiones.

Tomados en conjunto, estos resultados indican que estos esfuerzos tienen efectos complejos que requieren mayor investigación, y ofrecen un apoyo a los modelos cognitivos que postulan la existencia de vínculos entre los fallos en el autocontrol de los pensamientos y las autovaloraciones negativas (Purdon y Clark, 2001). Además de ello, los resultados avalan la utilidad de las estrategias terapéuticas cognitivas para el tratamiento del TOC, a través de las cuales se intenta convencer al paciente de que dejar fluir sus obsesiones no tiene consecuencias negativas, y que las diferencias entre intrusiones normales y obsesiones no se basan en sus respectivos contenidos, sino más bien en las valoraciones que se realizan de los mismos.

En conclusión, nuestros resultados muestran que los esfuerzos intencionados por suprimir pensamientos intrusos no agradables, generan más consecuencias negativas a los individuos que los esfuerzos por suprimir pensamientos neutros: existen más posibilidades de que se produzca un efecto rebote, y de que este efecto incremente la valoración de molestia y culpa asociada con el pensamiento. Asimismo, los esfuerzos de supresión deliberada interfieren con el proceso de habituación natural al pensamiento. En nuestra opinión, es necesario continuar con esta línea de investigación, dadas sus importantes implicaciones en la comprensión y el tratamiento del TOC.

\section{REFERENCIAS BIBLIOGRÁFICAS}

Abramowitz, J.S., Tolin, D.F., y Street, G.P. (2001). Paradoxical effects of thought suppression: A meta-analysis of controlled studies. Clinical Psychology Review, 21, 683-703.

Beck, A.T., Rush, A.J., Shaw, B.F., y Emery, G. (1979). Cognitive Therapy of Depression. New York: Guilford.

Clark, D.A., y Purdon, C.L. (1995). The assessment of unwanted intrusive thoughts: A review and critique of the literature. Behaviour Research and Therapy, 33, 967-976.

Clark, D.M., Ball, S., y Pape, D. (1991). An experimental investigation of thought suppression. Behaviour Research and Therapy, 29, 253-257.

Clark, D.M., Winton, E., y Thynn, L. (1993). A further experimental investigation of thought suppression. Behaviour Research and Therapy, 31, 207-210.

Freeston, M.H., y Ladouceur, R. (1993). Appraisal of cognitive intrusions and response style: Replication and extension. Behaviour Research and Therapy, 31, 181-190

Freeston, M.H., Ladouceur, R., Gagnon, F., y Thibodeau, N. (1991a). Cognitive intru- 
sions in a non-clinical population. I. Response style, subjective experience and appraisal. Behaviour Research and Therapy, 29, 585-597.

Freeston, M.H., Ladouceur, R., Provencher, M., y Blais, F. (1995). Strategies used with intrusive thoughts: context, appraisal, mood and efficacy. Journal of Anxiety Disorders, 9, 201-215.

Hodgson, R.J., y Rachman, S. (1977). Obsessive-compulsive complaints. Behaviour Research and Therapy, 15, 389-395.

Janeck, A.S., y Calamari, J.E. (1999). Thought suppression in obsessive-compulsive disorder. Cognitive Therapy and Research, 23, 497-509.

Kelly, A.E., y Kahn, J.H. (1994). Effects of suppression of personal intrusive thoughts. Journal of Personality and Social Psychology, 6, 998-1006.

Mc Nally, R.J., y Richiardi, J.N. (1996). Suppression of negative and neutral thoughts. Behavioral and Cognitive Psychotherapy, 24, 17-25.

Merckelbach, H., Muris, P., van den Hout, M., y DeJong, P. (1991). Rebound effects of thought suppression: instruction dependent?. Behavioral Psychotherapy, 19, 225 238.

Muris, P., Merckelbah, H., y De Jong, P. (1993). Verbalization and environmental cuing in thought suppression. Behaviour Research and Therapy, 35, 769-774.

Muris, P., Merckelbach, H., y Horselenberg, R. (1996). Individual differences in thought suppression. The White Bear Suppression Inventory: Factor structure, reliability, validity and correlates. Behaviour Research and Therapy, 34, 501-513.

Purdon, C. (1999). Thought suppression and psychopathology. Behaviour Research and Therapy, 37, 1029-1054.

Purdon, C y Clark, D. (1993). Obsessive intrusive thoughts in nonclinical subjects. Part I. Content and relation with depressive, anxious and obsessional symptoms. Behaviour Research and Therapy, 31, 713-720.

Purdon, C y Clark, D. (1994a). Obsessive intrusive thoughts in nonclinical subjects. Part II. Cognitive appraisal, emotional response and thought control strategies. Behaviour Research and Therapy, 32, 403-410.
Purdon, C., y Clark, D. (1994b). Perceived control and appraisal of obsessional thoughts: A replication and extension. Behavioral and Cognitive Psychotherapy, 22, 269-285.

Purdon, C., y Clark, D.A. (1999). Metacognition and obsessions. Clinical Psychology and Psychotherapy, 6, 102-110.

Purdon, C., y Clark, D.A. (2000). White bears and other elusive intrusions: assessing the relevance of thought suppression for obsessional problems. Behavior Modification, 24, 425-453.

Purdon, C., y Clark, D.A. (2001). Suppression of obsession-like thoughts in nonclinical individuals: impact on thought frequency, appraisal, and mood state. Behaviour Research and Therapy, 39, 1163-1181.

Rachman, S. (1997). A cognitive theory of obsessions. Behaviour Research and Therapy, 35, 793-802.

Rachman, S. (1998). A cognitive theory of obsessions: elaborations. Behaviour Research and Therapy, 36, 385-401.

Roemer, L., y Borkovec, T.D. (1994). Effects of suppressing thoughts about emotional material. Journal of Abnormal Psychology, 103, 467-474.

Rutledge, P.C. (1998). Obsessionality and the attempted suppression of unpleasant personal intrusive thoughts. Behaviour Research and Therapy, 36, 403-416.

Rutledge, P.C., Hancock, R.A., y Rutledge, J.H. (1996). Predictors of thought rebound. Behaviour Research and Therapy, 34, 555562.

Salkovskis, P.M. (1985). Obsessional-compulsive problems: A cognitive-behavioral analysis. Behaviour Research and Therapy, 23, 571-583.

Salkovskis, P.M. (1989). Cognitive-behavioral factors and the persistence of intrusive thoughts in obsessional problems. Behaviour Research and Therapy, 27, 149-160.

Salkovskis, P.M., y Campbell, P. (1994). Thought suppression induces intrusion in naturally occurring negative intrusive thoughts. Behaviour Research and Therapy, 32, 1-8.

Salkovskis, P.M., Richards, H.C., y Forrester, E. (1995). The relationship between obsessional problems and intrusive thoughts. 
Behavioral and Cognitive Psychotherapy, 23, 281-299.

Smári, J., Birgisdóttir, A.B., y Brynjólfsdóttir, B. (1995). Obsessive compulsive symptoms and suppression of personally relevant unwanted thoughts. Personality and Individual Differences, 18, 621-625.

Smári, J., Sigurjónsdóttir, H., y Saemundsdóttir, I. (1994). Thought suppression and obsession-compulsion. Psychological Reports, 75, 227-235.

Spielberger, C.D., Gorsuch, R.L., Lushene, R., Vagg, P.R., y Jacobs, G.A. (1983). Manual for the State-Trait Anxiety Inventory (form
Y). Palo Alto, CA: Consulting Psychologists Press, Inc.

Trinder, H., y Salkovkis, P.M. (1994). Personally relevant intrusions outside the laboratory: long term suppresion increases intrusion. Behaviour Research and Therapy, 32, 833-842.

Wegner, D.M. (1994). Ironic processes of mental control. Psychological Review, 101, 3452.

Wegner, D.M., Schneider, D.J., Carter, S.R., y White, T.L. (1987). Paradoxical effects of thought suppression. Journal of Personality and Social Psychology, 53, 5-13. 